\title{
Exploring the Origins of Enthalpy-Entropy Compensation by Calorimetric Studies of Cyclodextrin Complexes
}

Christian Schönbeck ${ }^{\mathrm{a}}$ and René Holmª

${ }^{a}$ Department of Science and Environment, Roskilde University, Universitetsvej 1, DK-4000, Roskilde, Denmark

${ }^{b}$ Drug Product Development, Janssen Research and Development, Johnson \& Johnson, Turnhoutseweg 30, 2340 Beerse, Belgium

Supporting Information 


\section{NMR spectra}

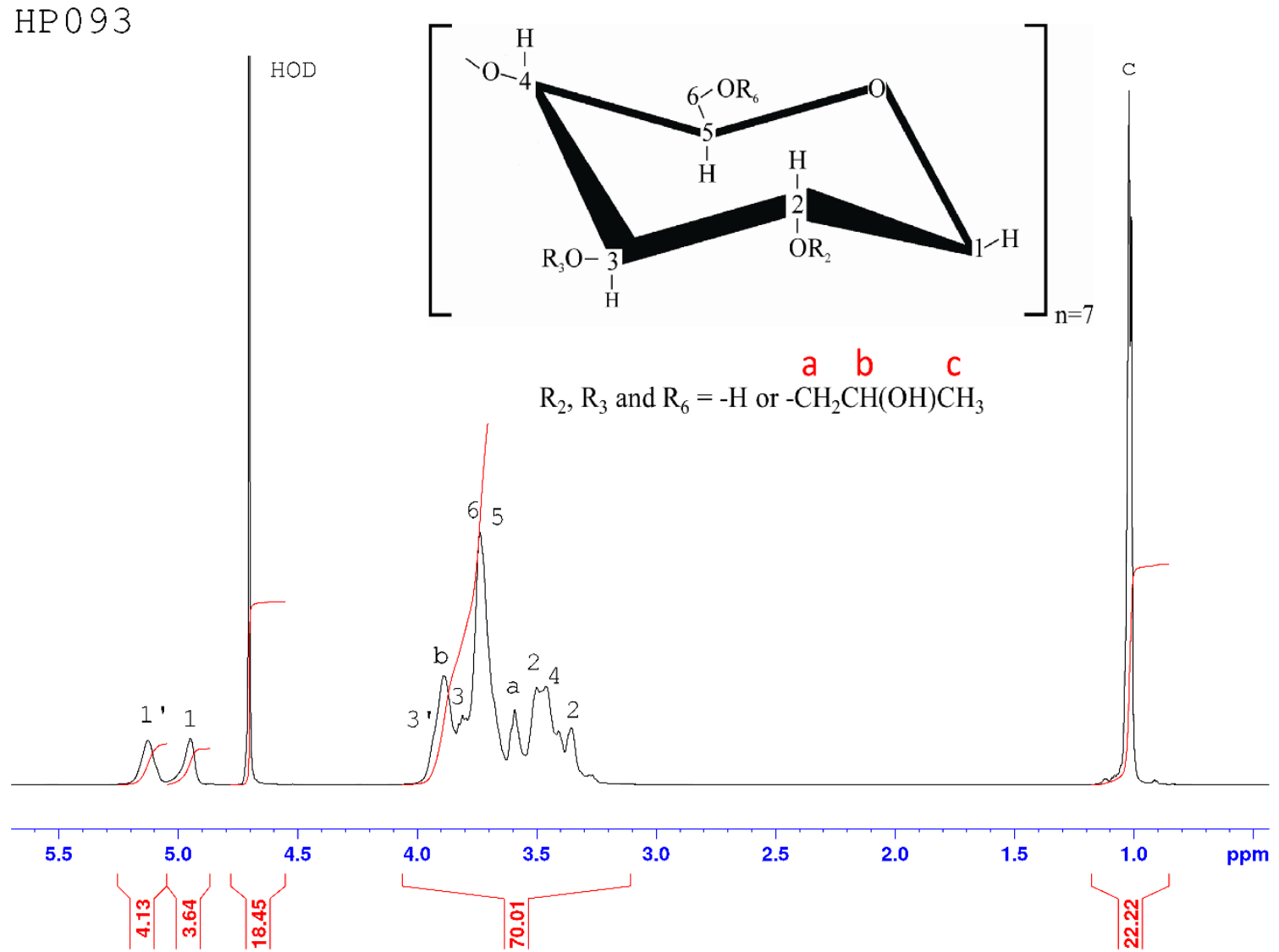

Figure $S 1{ }^{1} H$-NMR spectrum of the hydroxypropylated $\beta C D$, HP093. As the cyclodextrin is partially substituted some nuclei are observed in duplicate, one in a substituted glucose unit and one in a non-substituted glucose unit. Protons next to a substituted site are denoted by an apostrophe, e.g. 1' when a hydroxypropyl chain is attached to O2. Please refer to 'Section 3.1: Characterization of cyclodextrins' in the Ph.D. dissertation by Christian Schönbeck for details regarding the annotation and the calculation of the degree of substitution. ${ }^{1}$ 

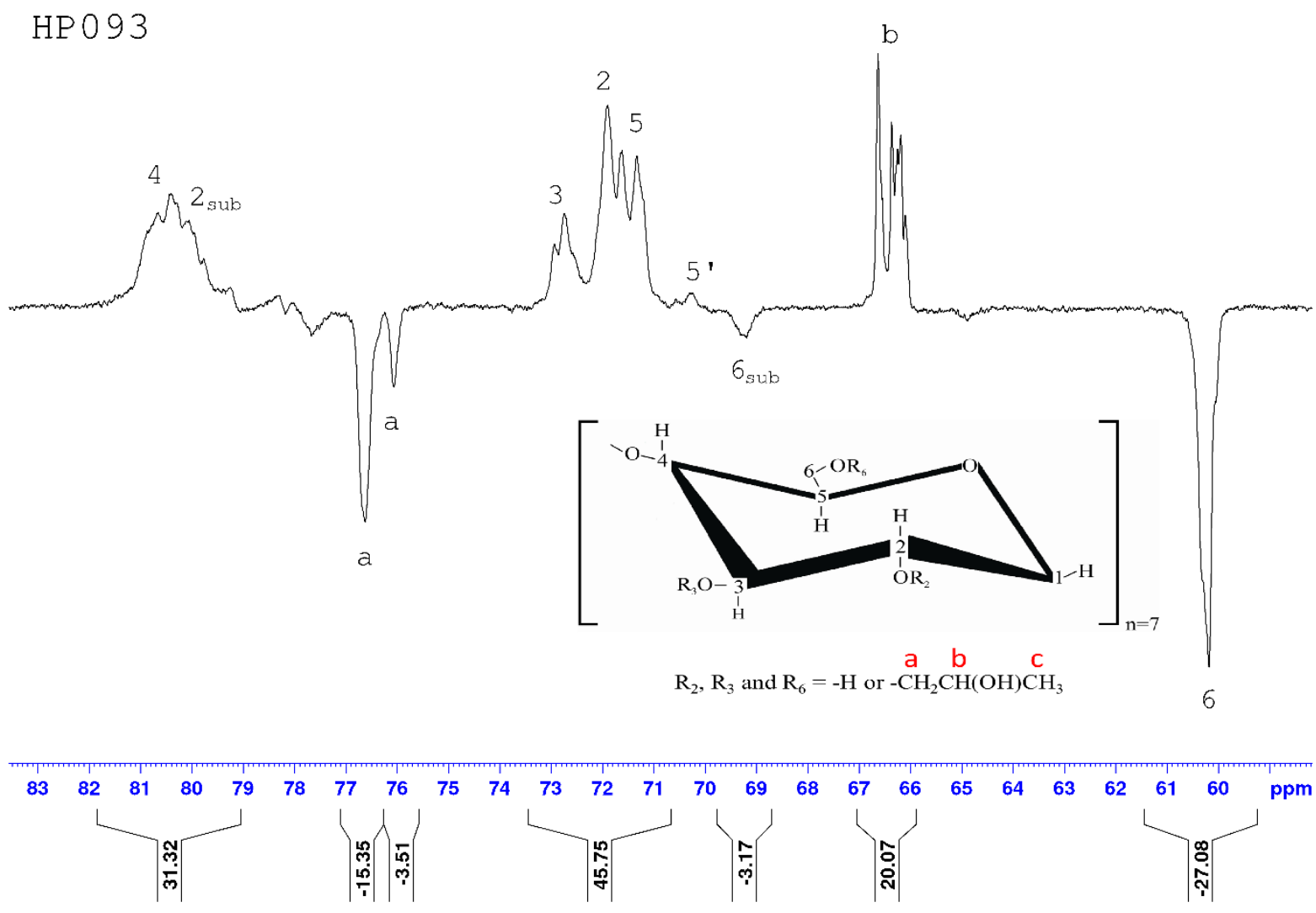

Figure $S 2{ }^{13} C$-NMR spectrum of the hydroxypropylated $\beta C D$, HP093. Positive signals are obtained for carbon atoms with one or three attached hydrogens while negative signals are obtained for carbons with zero or two attached hydrogens. As the cyclodextrin is partially substituted some nuclei are observed in duplicate, one in a substituted glucose unit and one in a non-substituted glucose unit. Carbons next to a substituted site are denoted by an apostrophe, e.g. 5' when a hydroxypropyl chain is attached to O6. Carbons directly attached to a substituted oxygen is denoted by the subscript 'sub, e.g. $\sigma_{\text {sub }}$ when a hydroxypropyl chain is attached to O6. The degree of substitution on $\mathrm{O6}$ is calculated from the areas of $\mathrm{C6}$ and $\mathrm{C6}_{\text {sub. }}$. Please refer to 'Section 3.1: Characterization of cyclodextrins' in the Ph.D. dissertation by Christian Schönbeck for details regarding the annotation. ${ }^{1}$ 


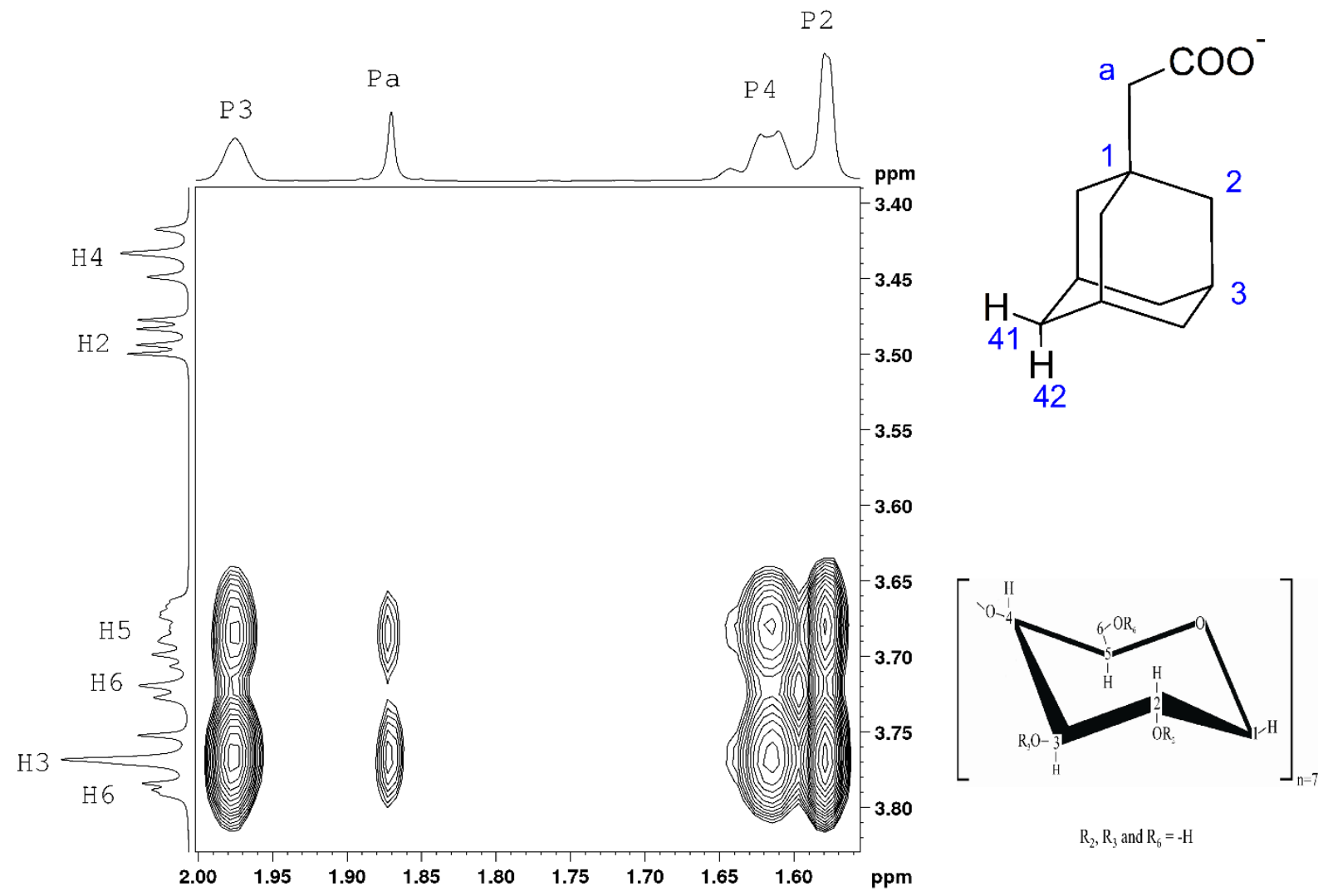

Figure S3 Partial ROESY spectrum of the $\beta C D$ : $A d C \mathrm{CH}_{2} \mathrm{COO}$ complex. Protons on the cyclodextrin host are denoted by ' $\mathrm{H}$ ' while protons on the adamantane derivative are denoted by ' $P$ '. Strong ROESY peaks are observed between all guest protons and the interior CD protons, $H 3$ and H5, thus confirming inclusion of the guest into the cavity of the CD. 


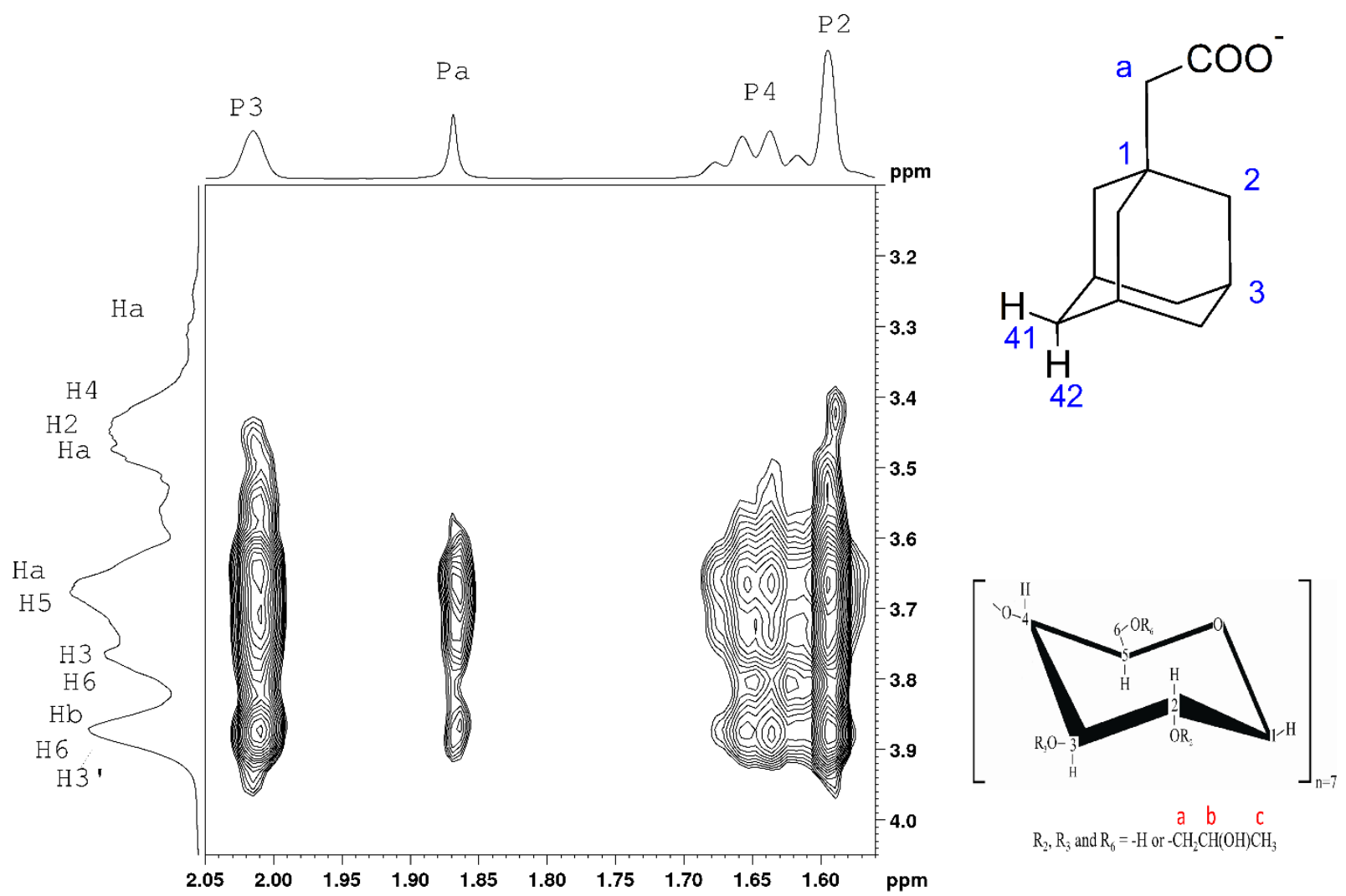

Figure S4 Partial ROESY spectrum of the HP093:AdCH $\mathrm{CHO}_{2} \mathrm{COmplex.}$ Protons on the cyclodextrin host are denoted by ' $\mathrm{H}$ ' while protons on the adamantane derivative are denoted by ' $P$ '. Annotation of the ROESY interactions are complicated by the broad and overlapping peaks, but the pattern seems quite similar to Figure S3 where the ROESY spectrum confirmed the inclusion of the adamantine derivative into the cavity of the cyclodextrin host. 


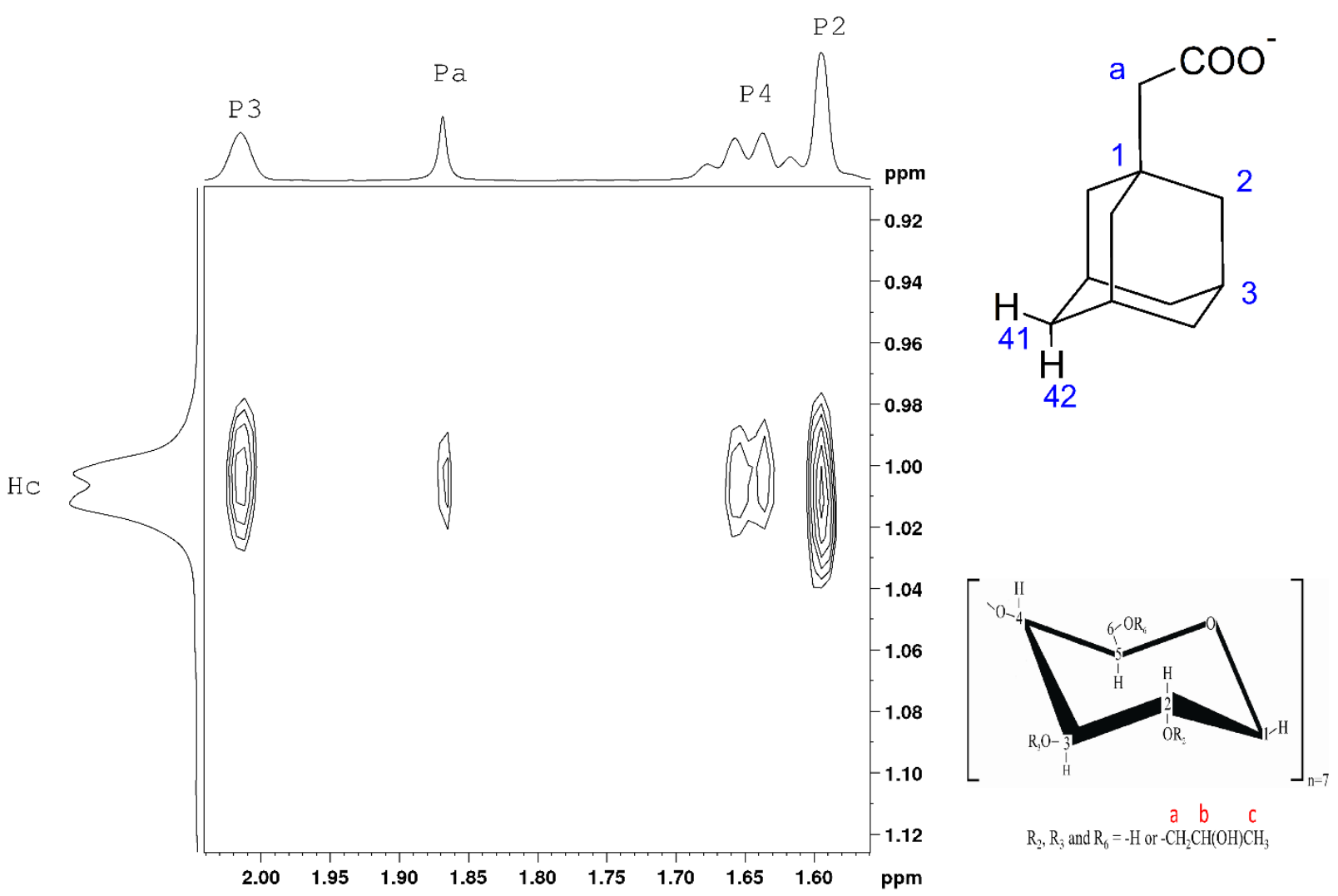

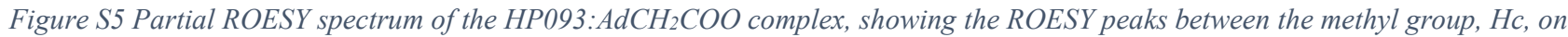
the hydroxypropyl chains of the cyclodextrin host and the protons on the adamantane derivative. The peaks are very weak compared to the peaks in Figure S4, indicating only limited interactions between hydroxypropyl chains and the adamantane derivative. 


\section{Data from MD simulations}

Table S1 Water accessible surface area (ASA) of the simulated complexes and free species. The areas are divided into polar (ASAp) and nonpolar (ASAnp) surface area. All values are in $A^{2}$.

\begin{tabular}{|c|c|c|c|c|c|c|}
\hline & \multirow{2}{*}{\multicolumn{2}{|c|}{ Free species }} & \multicolumn{4}{|c|}{ CD complexed with AdCOO } \\
\hline & & & \multicolumn{2}{|c|}{ Secondary complex } & \multicolumn{2}{|c|}{ Primary complex } \\
\hline & $\mathrm{ASA}_{\mathrm{p}}$ & $\mathrm{ASA}_{\mathrm{np}}$ & $\mathrm{ASA}_{\mathrm{p}}$ & $\mathrm{ASA}_{n p}$ & $\mathrm{ASA}_{\mathrm{p}}$ & $\mathrm{ASA}_{\mathrm{np}}$ \\
\hline$\beta C D$ & 685 & 470 & 691 & 415 & 628 & 431 \\
\hline HP086 & 648 & 956 & 647 & 891 & 620 & 900 \\
\hline \multirow[t]{3}{*}{ AdCOO } & 74 & 251 & - & - & - & - \\
\hline & & & \multicolumn{4}{|c|}{$\mathrm{CD}$ complexed with $\mathrm{AdCH}_{2} \mathrm{COO}$} \\
\hline & & & \multicolumn{2}{|c|}{ Secondary complex } & \multicolumn{2}{|c|}{ Primary complex } \\
\hline$\beta C D$ & 685 & 470 & 685 & 429 & 631 & 429 \\
\hline HP086 & 648 & 956 & 659 & 893 & 604 & 904 \\
\hline $\mathrm{AdCH}_{2} \mathrm{COO}$ & 77 & 270 & - & - & - & - \\
\hline
\end{tabular}




\section{Data from Isothermal Titration Calorimetry}

Table S2 Thermodynamic parameters at $25{ }^{\circ} \mathrm{C}$ for the binding of bile salts and adamantane derivatives to natural and hydroxypropylated B-cyclodextrins. Data for complexes with the three BSs are from previous publications. ${ }^{2-4}$ Cyclodextrins are named according to the average number of hydroxypropyl substituents per glucose unit, i.e. HP063 contains 0.63 substituents per glucose unit or $0.63 * 7=4.41$ substituents per cyclodextrin molecule.

\begin{tabular}{|c|c|c|c|c|c|c|}
\hline $\begin{array}{c}\text { Guest } \\
\text { molecule }\end{array}$ & Cyclodextrin & $\begin{array}{c}\mathrm{K} \\
\left(\mathrm{M}^{-1}\right)\end{array}$ & $\begin{array}{c}\Delta \mathrm{G} \\
(\mathrm{kJ} / \mathrm{mol})\end{array}$ & $\begin{array}{c}\Delta \mathrm{H} \\
(\mathrm{kJ} / \mathrm{mol})\end{array}$ & $\begin{array}{c}\mathrm{T} \Delta \mathrm{S} \\
(\mathrm{kJ} / \mathrm{mol})\end{array}$ & $\begin{array}{c}\Delta \mathrm{C}_{\mathrm{p}} \\
(\mathrm{J} / \mathrm{mol} / \mathrm{K})\end{array}$ \\
\hline \multirow[t]{3}{*}{$\mathrm{AdCOO}$} & $\beta C D$ & 38380 & -26.16 & -22.36 & 3.80 & -329 \\
\hline & HP063 & 9289 & -22.65 & -14.94 & 7.71 & -422 \\
\hline & НР093 & 4523 & -20.86 & -10.97 & 9.89 & -472 \\
\hline \multirow[t]{3}{*}{$\mathrm{AdCH}_{2} \mathrm{COO}$} & $\beta C D$ & 109789 & -28.77 & -23.52 & 5.25 & -374 \\
\hline & HP063 & 20428 & -24.60 & -15.28 & 9.32 & -453 \\
\hline & НР093 & 9622 & -22.74 & -10.19 & 12.55 & -524 \\
\hline \multirow[t]{3}{*}{$\mathrm{AdNH}_{3}$} & $\beta C D$ & 7159 & -22.00 & -20.07 & 1.93 & -311 \\
\hline & HP063 & 1579 & -18.26 & -12.89 & 5.37 & -341 \\
\hline & HP093 & 981 & -17.08 & -8.53 & 8.55 & -319 \\
\hline \multirow[t]{3}{*}{$\mathrm{AdOH}$} & $\beta C D$ & 42526 & -26.42 & -22.58 & 3.84 & -350 \\
\hline & HP063 & 12839 & -23.45 & -13.29 & 10.16 & -432 \\
\hline & НР093 & 7196 & -22.02 & -8.76 & 13.26 & -455 \\
\hline \multirow[t]{3}{*}{$\mathrm{AdCH}_{2} \mathrm{OH}$} & $\beta C D$ & 173091 & -29.90 & -27.77 & 2.13 & -391 \\
\hline & HР063 & 38955 & -26.20 & -17.48 & 8.72 & -447 \\
\hline & НР093 & 21258 & -24.70 & -12.39 & 12.31 & -497 \\
\hline \multirow[t]{6}{*}{ GC } & $\beta C D$ & 2960 & -19.81 & -25.2 & -5.4 & -318 \\
\hline & HP054 & 2141 & -19.01 & -13.6 & 5.4 & - \\
\hline & HР063 & 2084 & -18.94 & -11.5 & 7.4 & -413 \\
\hline & HP082 & 1229 & -17.63 & -6.8 & 10.8 & - \\
\hline & HP102 & 1162 & -17.50 & -3.5 & 14.0 & -494 \\
\hline & HP106 & 669 & -16.13 & -4.3 & 11.8 & - \\
\hline \multirow[t]{6}{*}{ GDC } & $\beta C D$ & 5673 & -21.43 & -28.5 & -7.1 & -271 \\
\hline & HP054 & 4326 & -20.75 & -16.3 & 4.5 & - \\
\hline & HP063 & 4677 & -20.95 & -13.3 & 7.6 & -458 \\
\hline & HP082 & 3284 & -20.07 & -7.8 & 12.3 & - \\
\hline & HP102 & 3348 & -20.12 & -4.2 & 15.9 & -574 \\
\hline & HP106 & 2405 & -19.30 & -4.0 & 15.3 & - \\
\hline \multirow[t]{6}{*}{ GCDC } & $\beta C D$ & 156,226 & -29.64 & -30.9 & -1.3 & -484 \\
\hline & HP054 & 84,657 & -28.13 & -22.4 & 5.7 & - \\
\hline & HР063 & 74,465 & -27.81 & -20.2 & 7.6 & -615 \\
\hline & HP082 & 41,935 & -26.38 & -16.1 & 10.3 & - \\
\hline & HP102 & 35,430 & -25.97 & -13 & 13.0 & -702 \\
\hline & HP106 & 22,617 & -24.85 & -12.8 & 12.1 & - \\
\hline
\end{tabular}




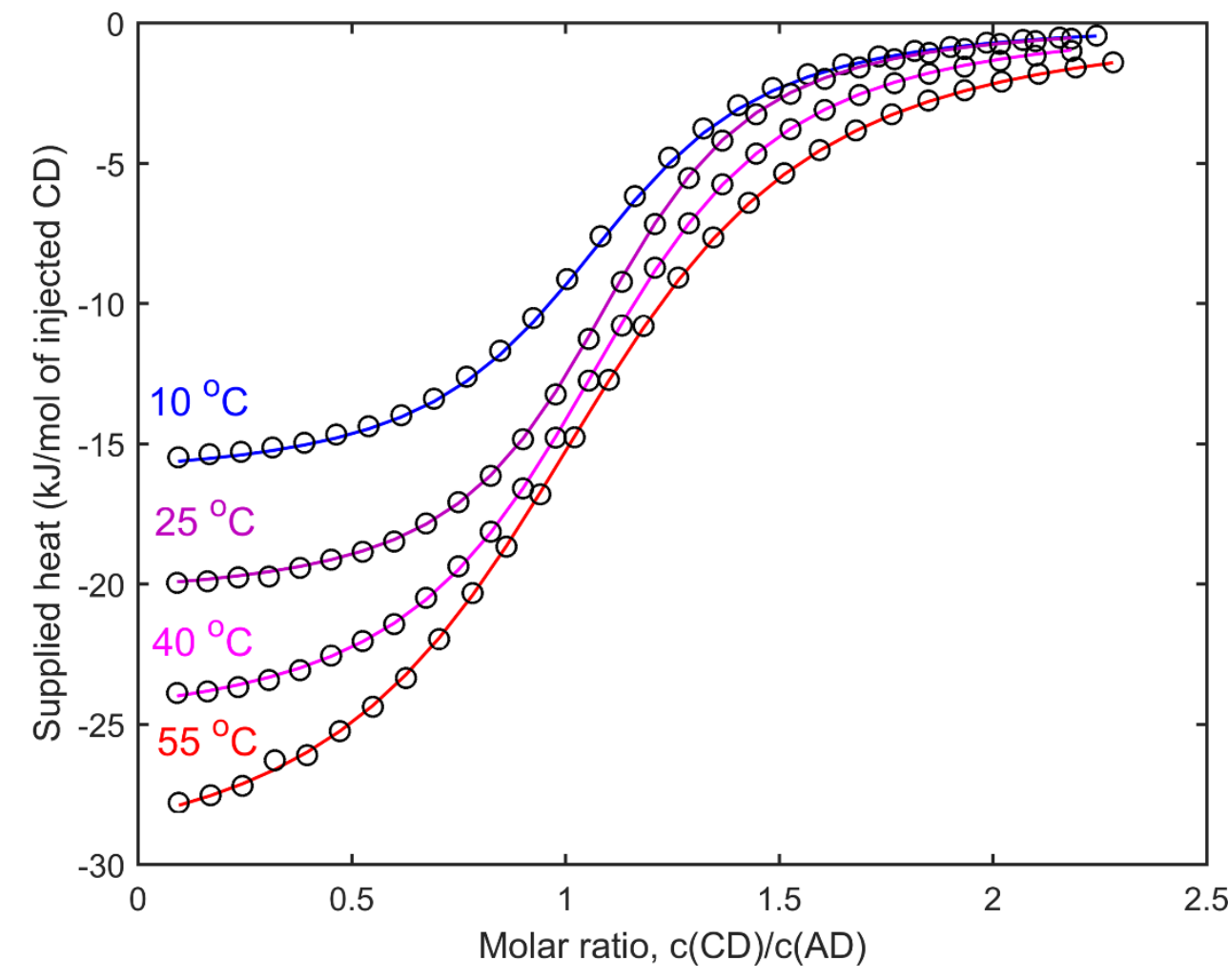

\begin{tabular}{lccccc}
\hline Parameter & $\begin{array}{c}\text { Best fit } \\
\text { value }\end{array}$ & $\begin{array}{c}\text { Error at } \\
\mathbf{9 5 \%} \mathbf{C L}\end{array}$ & \multicolumn{2}{c}{ Correlation Matrix } \\
\hline $\mathrm{K}$ at $25^{\circ} \mathrm{C}\left(\mathrm{M}^{-1}\right)$ & 38380 & 442 & 1 & 0.37 & 0.26 \\
$\Delta \mathrm{H}$ at $25^{\circ} \mathrm{C}(\mathrm{kJ} / \mathrm{mol})$ & -22.39 & 0.04 & & 1 & -0.43 \\
$\Delta \mathrm{C}_{\mathrm{p}}(\mathrm{J} / \mathrm{mol} / \mathrm{K})$ & -329 & 2 & & & 1 \\
\hline
\end{tabular}

Figure S6 Global fit of 4 calorimetric titrations of $A d C O O$ with $\beta C D$, conducted at 10, 25, 40 and $55^{\circ} \mathrm{C}$. The fitting parameter, $N$, was local and assumed the values 0.942, 0.943, 0.938, and 0.936 . 


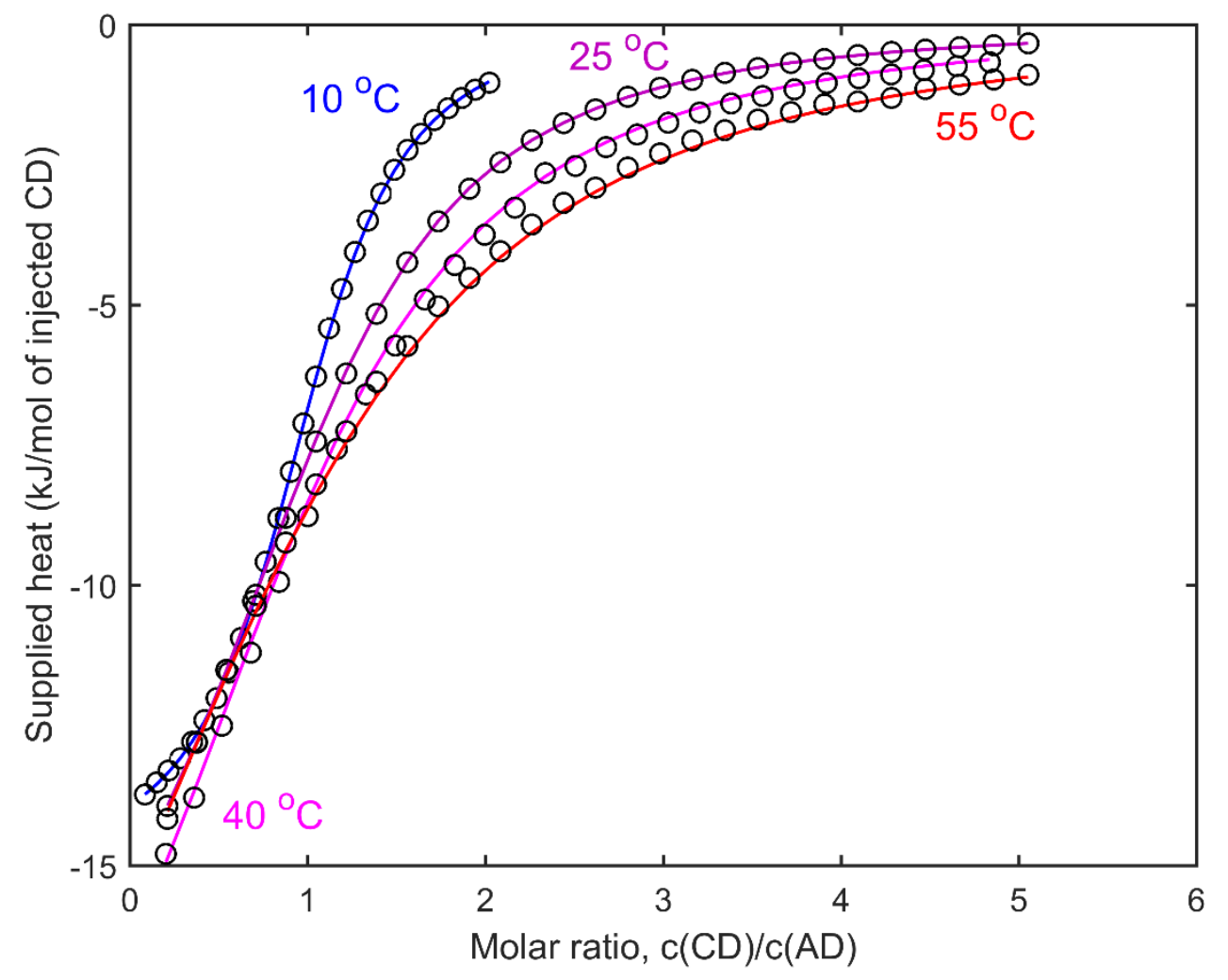

\begin{tabular}{lccccc}
\hline Parameter & $\begin{array}{c}\text { Best fit } \\
\text { value }\end{array}$ & $\begin{array}{c}\text { Error at } \\
\mathbf{9 5 \%} \text { CL }\end{array}$ & \multicolumn{2}{c}{ Correlation Matrix } \\
\hline $\mathrm{K}$ at $25^{\circ} \mathrm{C}\left(\mathrm{M}^{-1}\right)$ & 7159 & 269 & 1 & 0.74 & 0.34 \\
$\Delta \mathrm{H}$ at $25^{\circ} \mathrm{C}(\mathrm{kJ} / \mathrm{mol})$ & -20.07 & 0.12 & 1 & 0.58 \\
$\Delta \mathrm{C}_{\mathrm{p}}(\mathrm{J} / \mathrm{mol} / \mathrm{K})$ & -311 & 6 & & & 1 \\
\hline
\end{tabular}

Figure S2 Global fit of 4 calorimetric titrations of $A d N H_{3}$ with $\beta C D$, conducted at 10, 25, 40 and $55^{\circ} \mathrm{C}$. The fitting parameter, $N$, was local and assumed the values $0.978,0.971,0.963$, and 0.920 . 


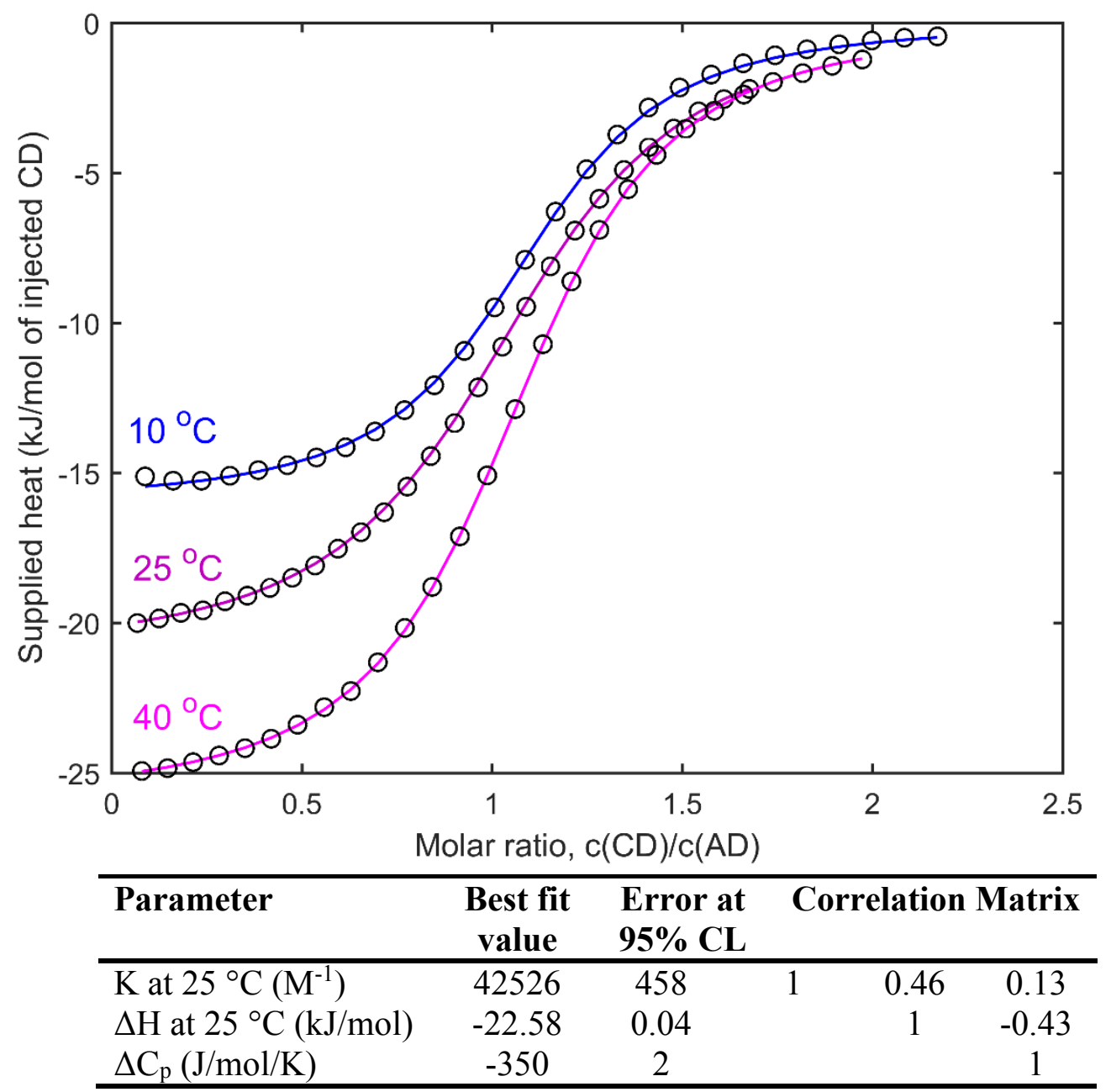

Figure S3 Global fit of 3 calorimetric titrations of $A d O H$ with $\beta C D$, conducted at $10,25,40{ }^{\circ} \mathrm{C}$. The fitting parameter, $N$, was local and assumed the values 0.921, 0.930, and 0.931. 


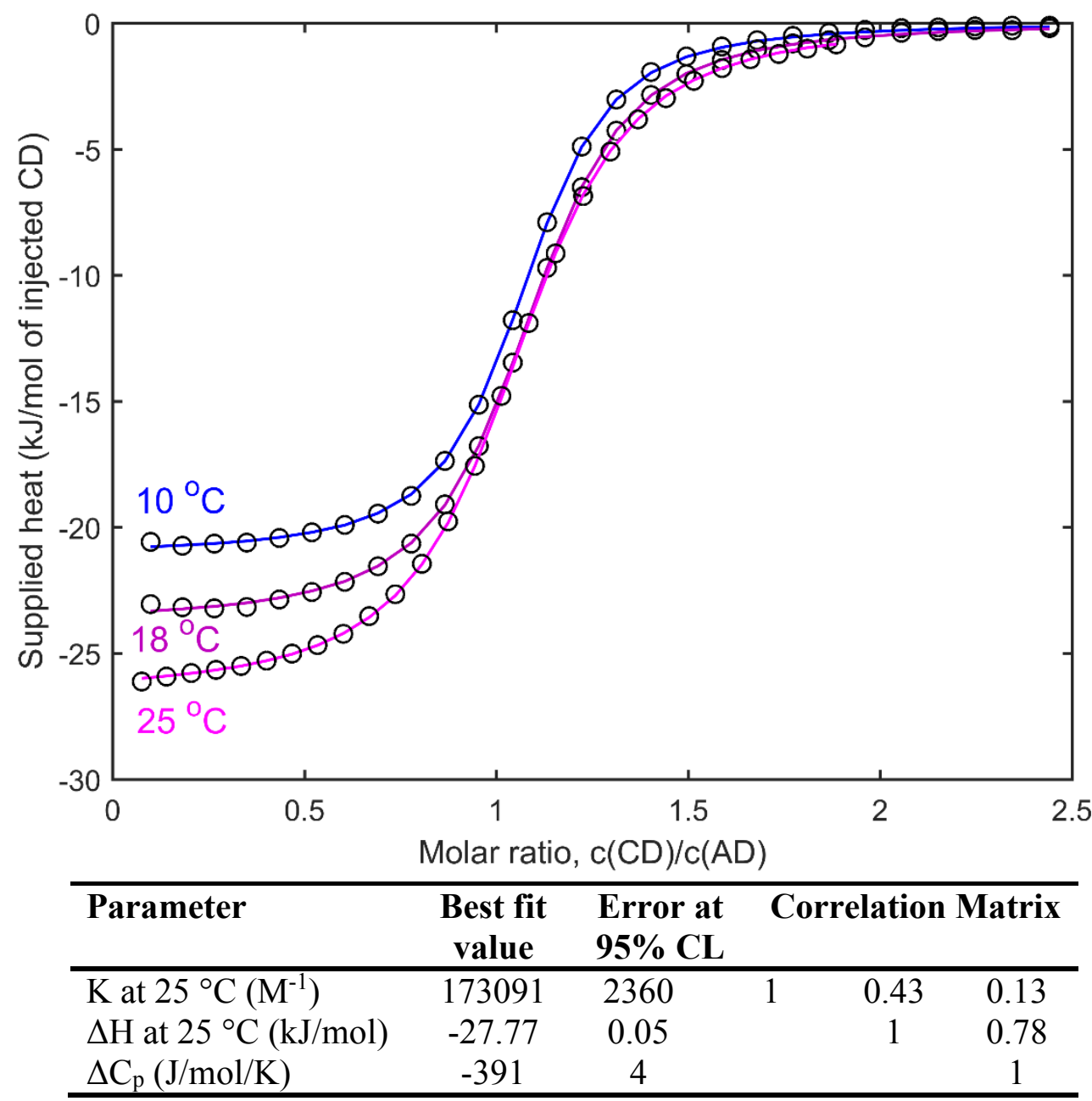

Figure $S 4$ Global fit of 3 calorimetric titrations of $\mathrm{AdCH} \mathrm{CH}_{2} \mathrm{OH}$ with $\beta C D$, conducted at 10,18 , and $25^{\circ} \mathrm{C}$. The fitting parameter, $\mathrm{N}$, was local and assumed the values 0.959, 0.945, and 0.9565 . 


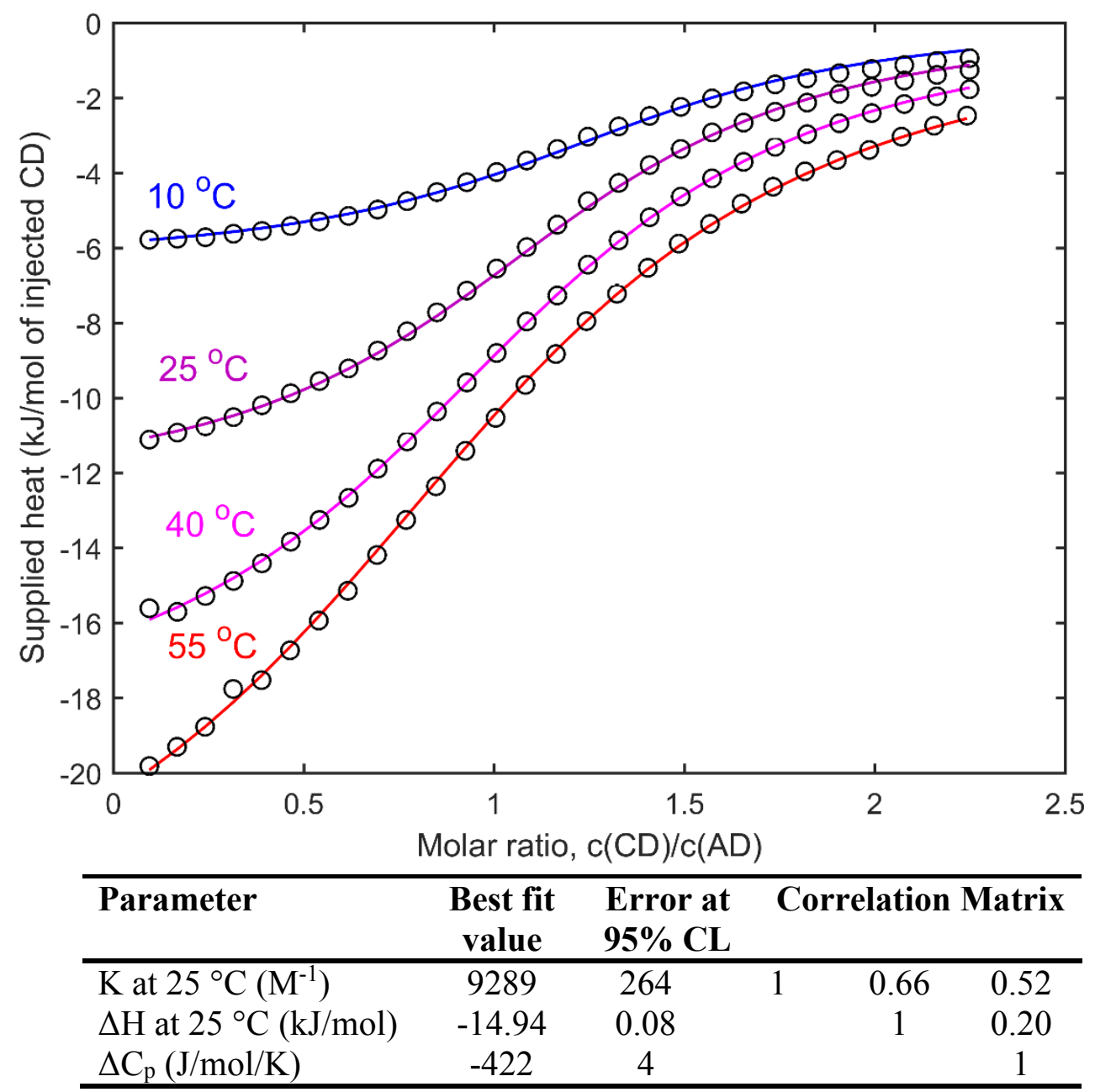

Figure S5 Global fit of 4 calorimetric titrations of AdCOO with HP063, conducted at 10, 25, 40 and $55{ }^{\circ} \mathrm{C}$. The fitting parameter, $N$, was local and assumed the values $0.736,0.832,0.860$, and 0.916. 


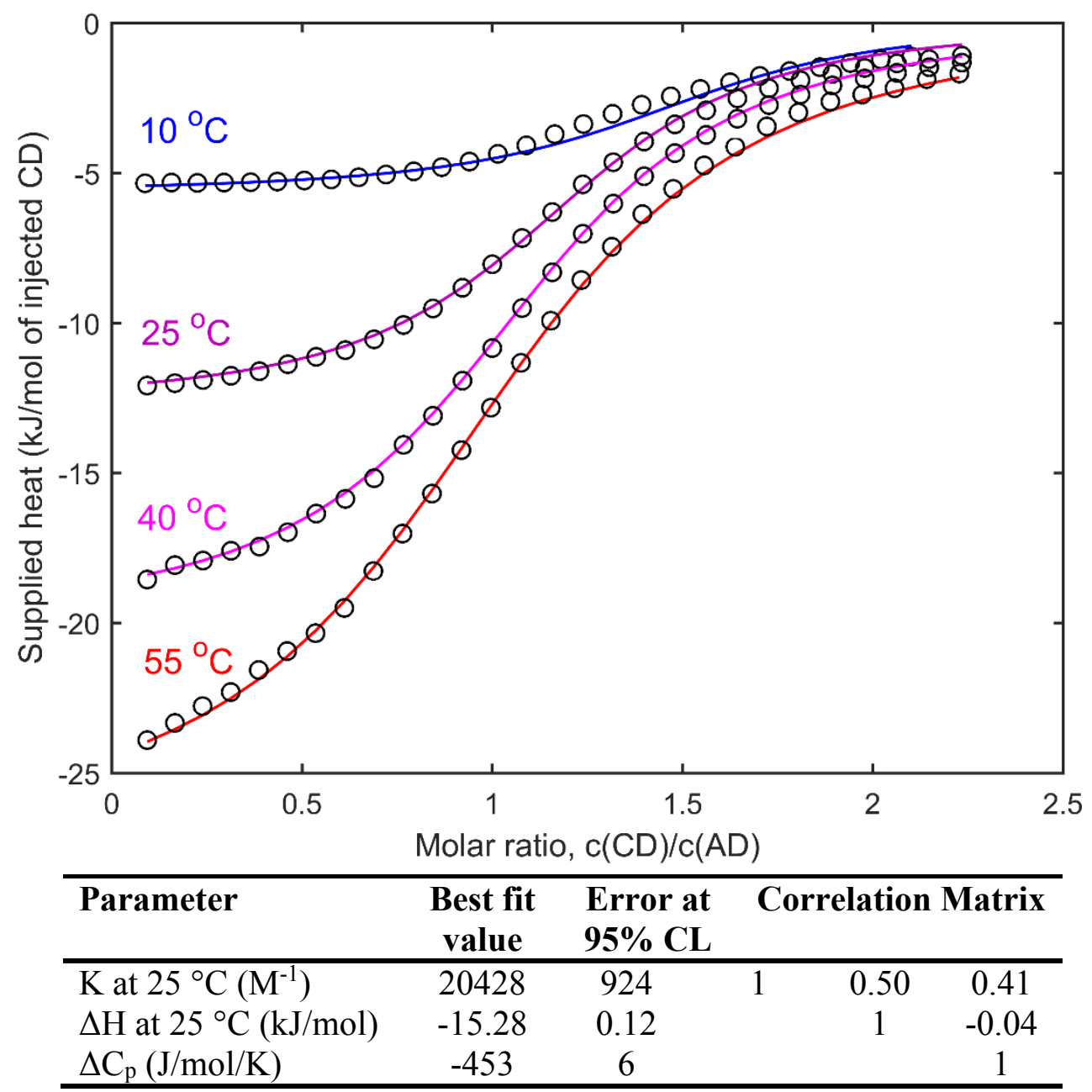

Figure S6 Global fit of 4 calorimetric titrations of $\mathrm{AdCH} \mathrm{CHOO}_{2}$ with $\mathrm{HPO63}$, conducted at 10, 25, 40 and $55^{\circ} \mathrm{C}$. The fitting parameter, $N$, was local and assumed the values $0.665,0.830,0.900$, and 0.935 . 


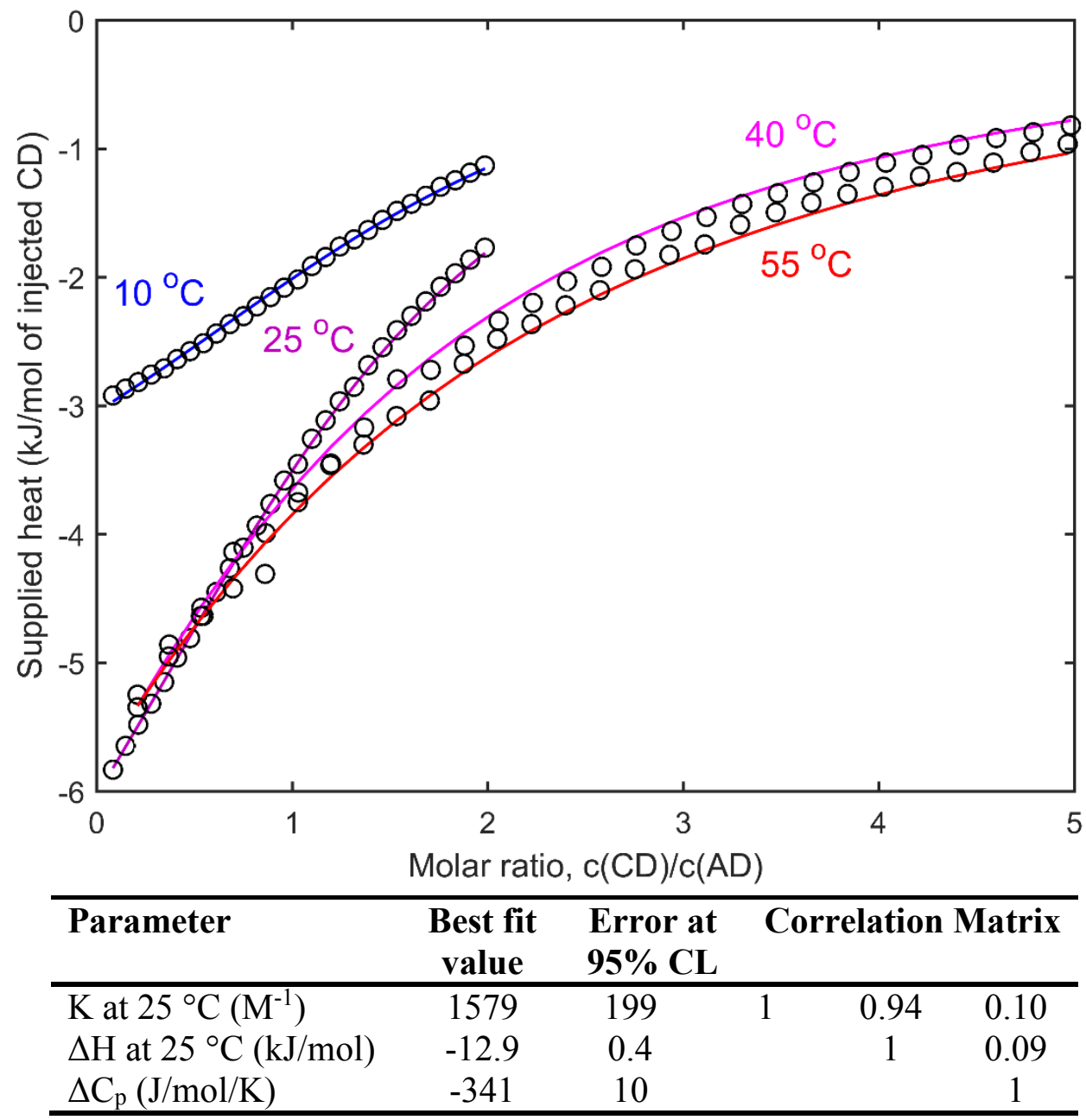

Figure $S 7$ Global fit of 4 calorimetric titrations of $A d N H 3$ with $H P 063$, conducted at 10, 25, 40 and $55^{\circ} \mathrm{C}$. The fitting parameter, $N$, was local and assumed the values 0.570, 0.733, 0.939, and 0.935 . 


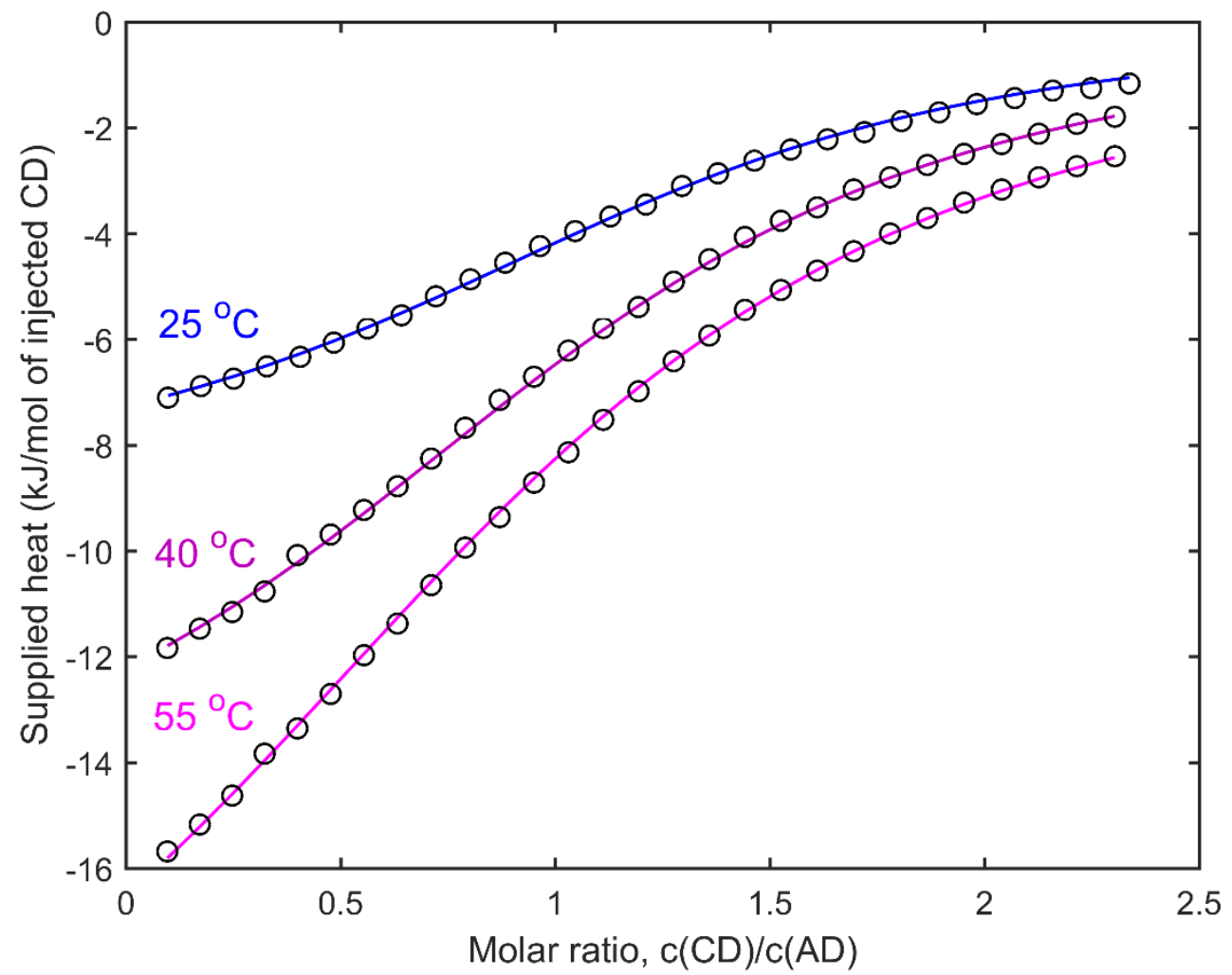

\begin{tabular}{lccccc}
\hline Parameter & $\begin{array}{c}\text { Best fit } \\
\text { value }\end{array}$ & $\begin{array}{c}\text { Error at } \\
\mathbf{9 5 \%} \mathbf{C L}\end{array}$ & \multicolumn{2}{c}{ Correlation Matrix } \\
\hline $\mathrm{K}$ at $25^{\circ} \mathrm{C}\left(\mathrm{M}^{-1}\right)$ & 4523 & 146 & 1 & 0.51 & 0.57 \\
$\Delta \mathrm{H}$ at $25^{\circ} \mathrm{C}(\mathrm{kJ} / \mathrm{mol})$ & -10.97 & 0.10 & & 1 & -0.20 \\
$\Delta \mathrm{C}_{\mathrm{p}}(\mathrm{J} / \mathrm{mol} / \mathrm{K})$ & -472 & 6 & & & 1 \\
\hline
\end{tabular}

Figure S8 Global fit of 3 calorimetric titrations of AdCOO with HP093, conducted at 25, 40 and $55{ }^{\circ} \mathrm{C}$. The fitting parameter, $N$, was local and assumed the values $0.807,0.873$, and 0.936 . 


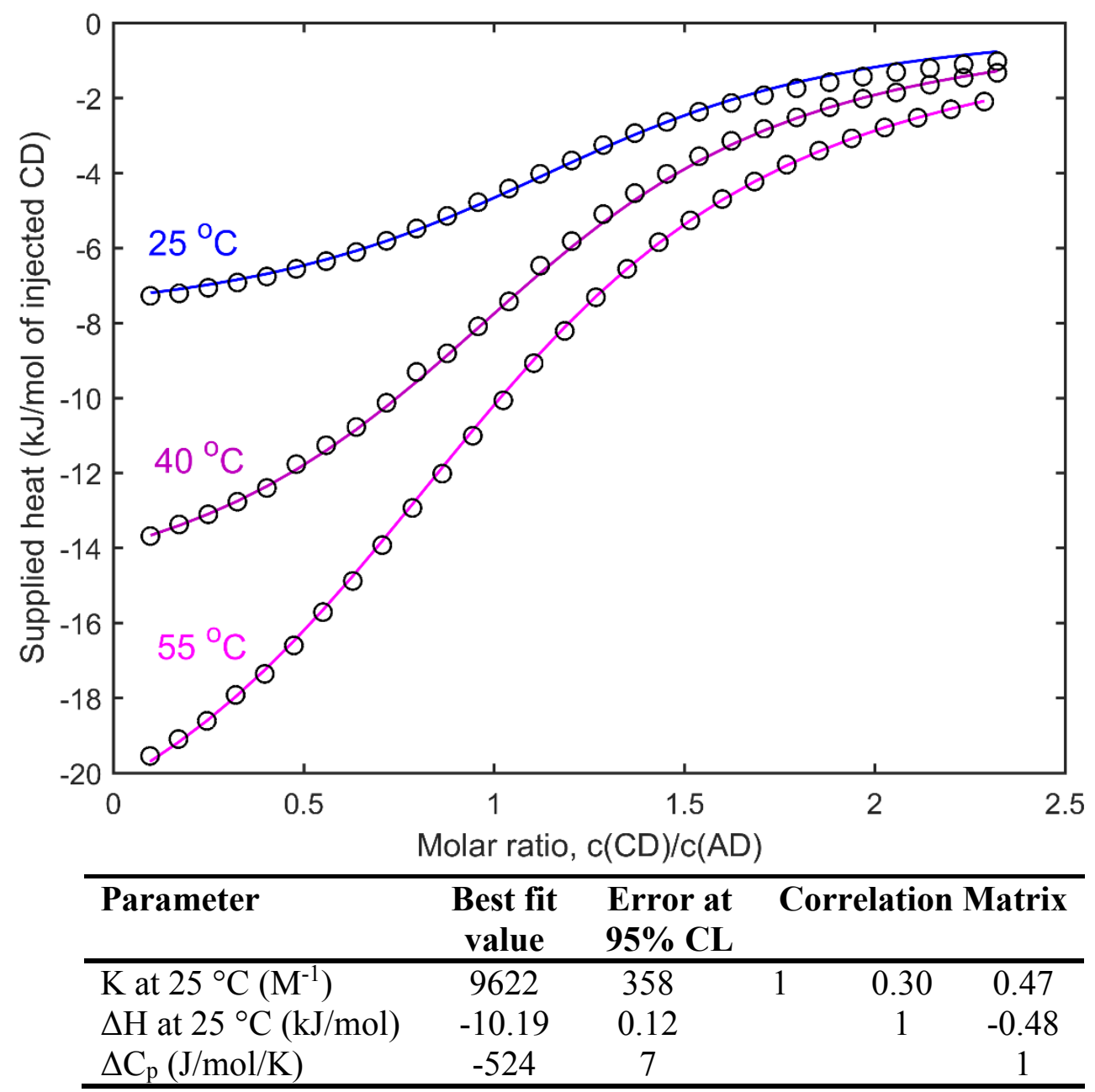

Figure S9 Global fit of 3 calorimetric titrations of $\mathrm{AdCH} \mathrm{CHOO}_{2}$ with $\mathrm{HP093}$, conducted at 25,40 and $55{ }^{\circ} \mathrm{C}$. The fitting parameter, $\mathrm{N}$, was local and assumed the values $0.785,0.875$, and 0.936 . 


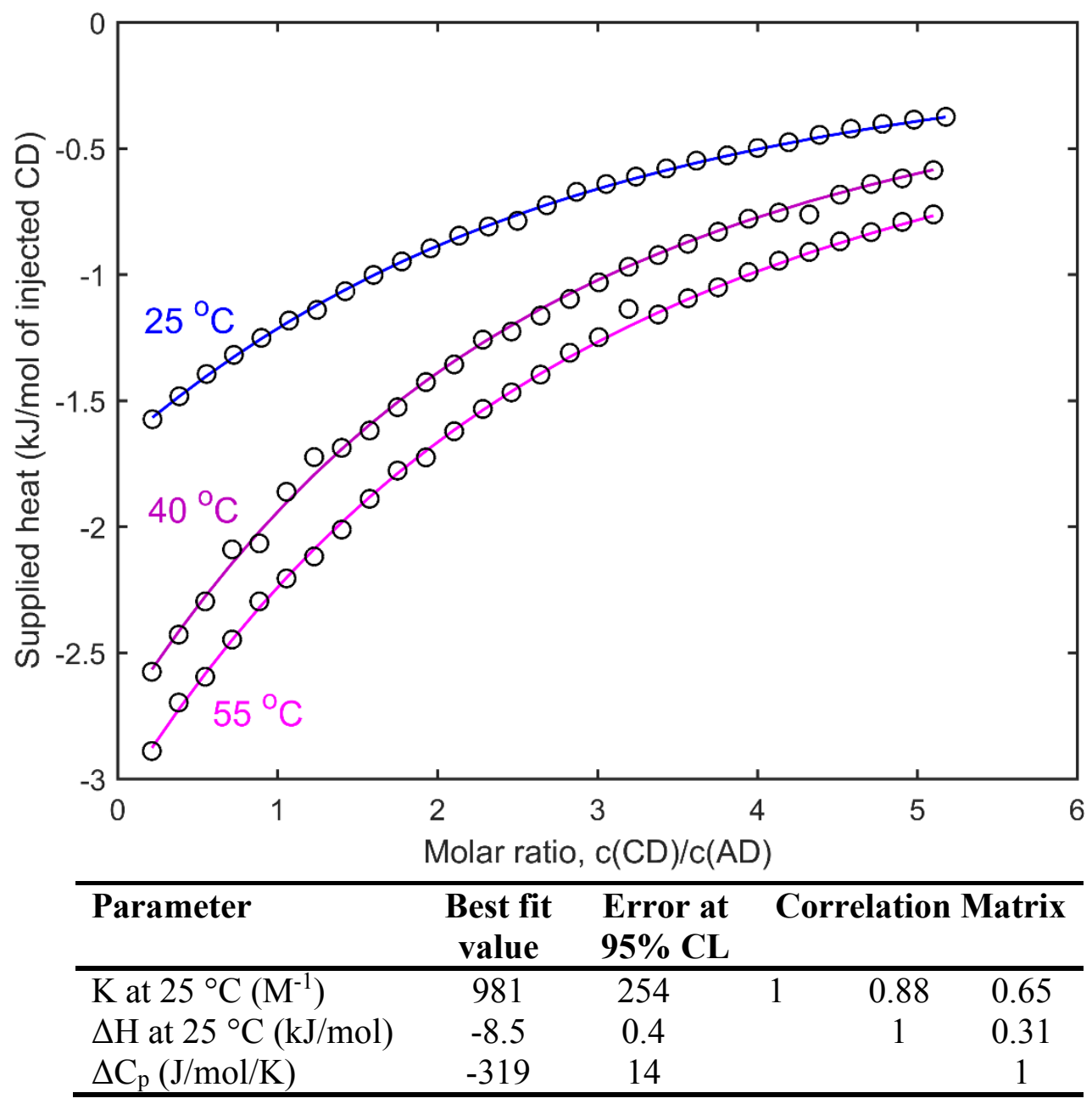

Figure S10 Global fit of 3 calorimetric titrations of $A d N H_{3}$ with HP093, conducted at 25, 40 and $55{ }^{\circ} \mathrm{C}$. The fitting parameter, $N$, was local and assumed the values 0.646, 0.792, 0.804 .

\begin{tabular}{lccccc}
\hline Parameter & $\begin{array}{c}\text { Best fit } \\
\text { value }\end{array}$ & $\begin{array}{c}\text { Error at } \\
\mathbf{9 5 \%} \text { CL }\end{array}$ & \multicolumn{2}{c}{ Correlation Matrix } \\
\hline $\mathrm{K}$ at $25^{\circ} \mathrm{C}\left(\mathrm{M}^{-1}\right)$ & 12839 & 373 & 1 & 0.57 & 0.49 \\
$\Delta \mathrm{H}$ at $25^{\circ} \mathrm{C}(\mathrm{kJ} / \mathrm{mol})$ & -13.29 & 0.07 & 1 & 0.04 \\
$\Delta \mathrm{C}_{\mathrm{p}}(\mathrm{J} / \mathrm{mol} / \mathrm{K})$ & -432 & 4 & & & 1 \\
\hline
\end{tabular}

Figure S11 Global fit of 4 calorimetric titrations of AdOH with HP063, conducted at 10, 25, 40 and $55^{\circ} \mathrm{C}$. The fitting parameter, $\mathrm{N}$, was local and assumed the values 0.737, 0.852, 0.907, and 0.927. 


\begin{tabular}{lccccc}
\hline Parameter & $\begin{array}{c}\text { Best fit } \\
\text { value }\end{array}$ & $\begin{array}{c}\text { Error at } \\
\mathbf{9 5 \%} \text { CL }\end{array}$ & Correlation Matrix \\
\hline $\mathrm{K}$ at $25^{\circ} \mathrm{C}\left(\mathrm{M}^{-1}\right)$ & 38955 & 1857 & 1 & 0.61 & 0.27 \\
$\Delta \mathrm{H}$ at $25^{\circ} \mathrm{C}(\mathrm{kJ} / \mathrm{mol})$ & -17.48 & 0.10 & & 1 & 0.21 \\
$\Delta \mathrm{C}_{\mathrm{p}}(\mathrm{J} / \mathrm{mol} / \mathrm{K})$ & -447 & 8 & & & 1 \\
\hline
\end{tabular}

Figure S12 Global fit of 5 calorimetric titrations of $\mathrm{AdCH}_{2} \mathrm{OH}$ with $\mathrm{HP063}$, conducted at 10, 17, 25, 32, and $40{ }^{\circ} \mathrm{C}$. The fitting parameter, $N$, was local and assumed the values 0.814, 0.870, 0.917, 0.937, and 0.961.

\begin{tabular}{lccccc}
\hline Parameter & $\begin{array}{c}\text { Best fit } \\
\text { value }\end{array}$ & $\begin{array}{c}\text { Error at } \\
\mathbf{9 5 \%} \mathbf{C L}\end{array}$ & \multicolumn{2}{c}{ Correlation Matrix } \\
\hline $\mathrm{K}$ at $25^{\circ} \mathrm{C}\left(\mathrm{M}^{-1}\right)$ & 7196 & 175 & 1 & 0.38 & 0.53 \\
$\Delta \mathrm{H}$ at $25^{\circ} \mathrm{C}(\mathrm{kJ} / \mathrm{mol})$ & -8.76 & 0.07 & & 1 & -0.37 \\
$\Delta \mathrm{C}_{\mathrm{p}}(\mathrm{J} / \mathrm{mol} / \mathrm{K})$ & -455 & 4 & & & 1 \\
\hline
\end{tabular}

Figure S13 Global fit of 3 calorimetric titrations of AdOH with HP093, conducted at 25, 40 and $55{ }^{\circ} \mathrm{C}$. The fitting parameter, $\mathrm{N}$, was local and assumed the values 0.798, 0.894, and 0.940 .

\begin{tabular}{lccccc}
\hline Parameter & $\begin{array}{c}\text { Best fit } \\
\text { value }\end{array}$ & $\begin{array}{c}\text { Error at } \\
\mathbf{9 5 \%} \mathbf{C L}\end{array}$ & \multicolumn{2}{c}{ Correlation Matrix } \\
\hline $\mathrm{K}$ at $25^{\circ} \mathrm{C}\left(\mathrm{M}^{-1}\right)$ & 21258 & 1133 & 1 & 0.71 & 0.30 \\
$\Delta \mathrm{H}$ at $25^{\circ} \mathrm{C}(\mathrm{kJ} / \mathrm{mol})$ & -12.39 & 0.09 & & 1 & 0.42 \\
$\Delta \mathrm{C}_{\mathrm{p}}(\mathrm{J} / \mathrm{mol} / \mathrm{K})$ & -497 & 9 & & & 1 \\
\hline
\end{tabular}

Figure $\mathrm{S} 14$ Global fit of 3 calorimetric titrations of $\mathrm{AdCH} \mathrm{CH}_{2} \mathrm{OH}$ with $\mathrm{HP093}$, conducted at 10, 17, 25, and $32{ }^{\circ} \mathrm{C}$. The fitting parameter, $N$, was local and assumed the values 0.616, 0.812, 0.885, and 0.928. 


\section{References}

(1) Schönbeck, C. Structure and Thermodynamics of Inclusion Complexes between Cyclodextrins and Bile Salts in Aqueous Solution, Roskilde University: Roskilde, 2012. (https://forskning.ruc.dk/en/publications/structure-and-thermodynamics-of-inclusioncomplexes-between-cyclo)

(2) Schönbeck, C.; Westh, P.; Madsen, J. C.; Larsen, K. L.; Städe, L. W.; Holm, R. Hydroxypropyl-Substituted $\beta$-Cyclodextrins: Influence of Degree of Substitution on the Thermodynamics of Complexation with Tauroconjugated and Glycoconjugated Bile Salts. Langmuir 2010, 26, 17949-17957.

(3) Schönbeck, C.; Holm, R.; Westh, P. Higher Order Inclusion Complexes and Secondary Interactions Studied by Global Analysis of Calorimetric Titrations. Anal. Chem. 2012, 84, 2305-2312.

(4) Schönbeck, C.; Holm, R.; Westh, P.; Peters, G. H. Extending the Hydrophobic Cavity of $\beta$ Cyclodextrin Results in More Negative Heat Capacity Changes but Reduced Binding Affinities. J. Incl. Phenom. Macrocycl. Chem. 2014, 78, 351-361. 